\title{
The Case of Squamous Cell Carcinoma Accompanied Leukemoid Reaction
} Z Urakçı, MA Kaplan, M Küçüköner, A Doğan, ZŞ Oruç, SB Zincircioğlu, E Bodakçi, N Akdeniz, A Işıkdoğan

\begin{abstract}
Leukemoid reaction is characterized by an elevation in neutrophil levels in peripheral blood and is defined as a total leukocyte number exceeding $50,000 / \mathrm{mm}^{3}$. An increase in mature neutrophils occurs in peripheral blood in Leukemoid reaction. A 70-year-old man was referred to us due to development of leukocytosis. His history revealed that the mass had begun beneath the armpit 2 months previously and had spread rapidly. At physical examination his general condition was poor, and he was conscious, oriented and cooperative. Yellow-colored, painful, ulcerated and necrotized masses with discharge, attached to the skin and approximately $16 \times 13 \mathrm{~cm}$ in size were determined in the left axilla. A painful, ulcerated, hemorrhagic mass with discharge attached to the skin and appearing to begin beneath the ear lobe, 10x9 $\mathrm{cm}$ in size, was determined below the ear. White cell count was $100,000 \mathrm{~mm}^{3}$. Ninety percent segmented neutrophils, $6 \%$ non-segmented neutrophils, $1.1 \%$ lymphocytes, $2.3 \%$ monocytes, $0.2 \%$ basophils and $0.3 \%$ eosinophils were determined at peripheral smear. Leukocyte alkaline phosphatase score was high. Squamous cell carcinoma with paraneoplastic Leukemoid reaction is an exceedingly rare combination. This case report was intended to remind physicians of the condition.
\end{abstract}

Keywords: Leukemoid reaction, leukocyte alkaline phosphatase, squamous cell carcinoma

From: ${ }^{1}$ Dicle University , Faculty of Medicine, Department of Medikal Oncology, Diyarbakir, Turkey, ${ }^{2}$ Dicle University Faculty of Medicine, Department of Internal Medicine, Diyarbakir, Turkey, and 3 Dicle University Faculty of Medicine, Department of Radiation Oncology, Diyarbakir, Turkey.

Correspondence: Dr A Doğan, Dicle University Faculty of Medicine, Department of Internal Medicine, Diyarbakir, Turkey. Fax: +904-122488523, e-mail: dr.a.dogan@ hotmail.com 


\section{INTRODUCTION}

Leukemoid reaction (LR) is characterized by an elevation in neutrophil levels in peripheral blood and is defined as a total leukocyte number exceeding $50,000 / \mathrm{mm}^{3}$. An increase in mature neutrophils occurs in peripheral blood in LR, but not in basophils. There are no blasts or nucleated erythrocytes. Leukocyte alkaline phosphatase (LAP) scores rise (1). The condition can be differentiated in this way from chronic myeloid leukemia with $\mathrm{t}(9-22)$ positivity and a normal LAP score. The most common cause of LR is infection, although other causes such as malignancy, severe hemorrhage, sudden hemolysis and drug use may be seen (2) Pulmonary nasopharyngeal GIS and GUS malignities are the most common malignities in LR (3).

The purpose of this case report is to remind physicians that squamous cell carcinoma (SCC) may sometimes accompany LR, albeit rarely.

\section{CASE REPORT}

A 70-year-old man was referred to us due to development of leukocytosis while being monitored for a mass below the armpit and the ear at an external health center. His history revealed that the mass had begun beneath the armpit 2 months previously and had spread rapidly. He had no previous history of tumoral development, and there was no history of similar symptoms in the family. At physical examination his general condition was poor, and he was conscious, oriented and cooperative. Body temperature was normal. Appearance was cachectic. Body temperature was $36.5 \mathrm{C}$ and arterial tension was 115/80. Yellow-colored, painful, ulcerated and necrotized masses with discharge, attached to the skin and approximately $16 \times 13 \mathrm{~cm}$ in size were determined in the left axilla (Figure 1). 


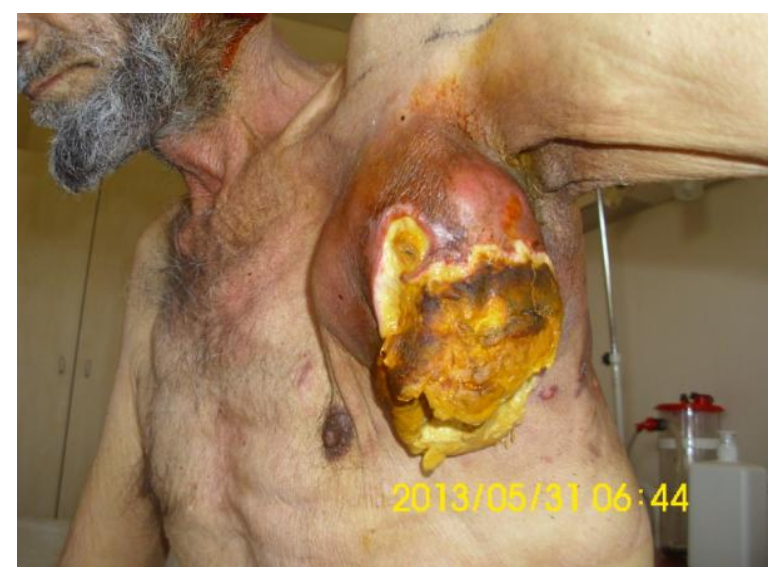

Fig.1: Huge tumor on his left axillary region

A painful, ulcerated, hemorrhagic mass with discharge attached to the skin and appearing to begin beneath the ear lobe, 10x9 $\mathrm{cm}$ in size, was determined below the ear (Figure 2).

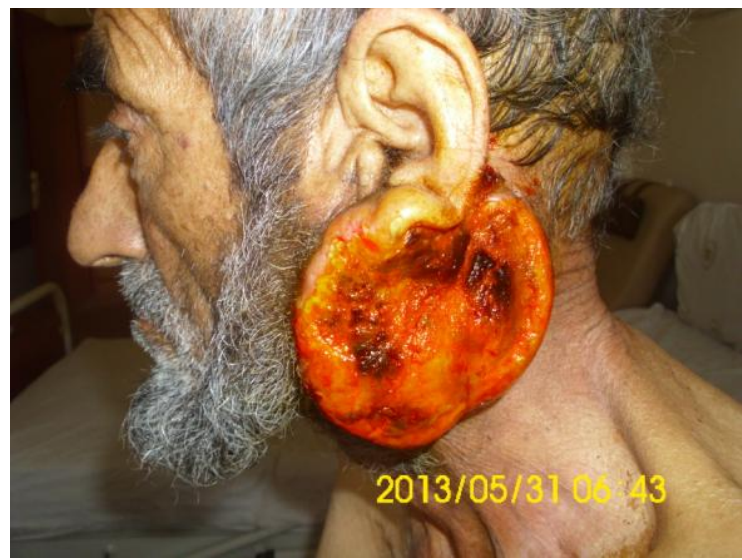

Fig.2: Ulcerated mass on his lower ear region

Cardiovascular, respiratory and gastrointestinal system examinations were normal. Laboratory values were hemoglobin $12.5 \mathrm{gr} / \mathrm{dL}$ and platelet number $210,000 \mathrm{~mm}^{3}$. Calcium level was $15.5 \mathrm{mg} / \mathrm{dL}$. Routine serum electrolytes and liver and kidney functions were normal. Creactive protein was $9 \mathrm{mg} / \mathrm{dl}$. White cell count was 100,000 $\mathrm{mm}^{3}$. Ninety percent segmented neutrophils, $6 \%$ non-segmented neutrophils, $1.1 \%$ lymphocytes, $2.3 \%$ monocytes, $0.2 \%$ basophils and $0.3 \%$ eosinophils were determined at peripheral smear. A picture of LR with 
neutrophil predominance was present at peripheral blood smear. Leukocyte alkaline phosphatase (LAP) score was high. Bone marrow biopsy was performed. Increased myeloid series and hypercellularity were determined. Cytogenetic analysis, pulmonary x-ray and abdominal CT were normal. Thoracic CT revealed air pockets invading the pectoral muscles in the left axillary region, a tumoral mass with wide necrotic components infiltrating the skin and occasional invasion in fat planes between the mass and the sub-scapularis muscle. In addition, enlarged lymph glands were present in the left retropectoral region. At abdominal CT, the appearance of the liver was compatible with adrenal gland metastasis. Surface biopsy was taken from the mass. Staphylococcus hominis grew in culture from the ulcerated, malodorous tumor site. C-protein returned to normal following antibiotherapy initiated on the basis of the culture antibiogram. Following exclusion of secondary causes, this picture was attributed to SCC-related paraneoplastic LR. Chemotherapy could not be administered due to low X performance. White cell count on the $21^{\text {st }}$ day of hospitalization was $127,000 / \mathrm{mm}^{3}$. (Neutrophil level at peripheral smear rose 93\%). Following debulking surgery, the patient was given radiotherapy directed toward the large mass. During monitoring, white cell count decreased to 15,000 . However, the patient developed pneumonia and died from acute respiratory dyspnea syndrome on the $5^{\text {th }}$ day of hospitalization.

\section{CONCLUSION}

LR refers to a leukocyte number above $50,000 / \mathrm{mm}^{3}$ in which neutrophils predominant in peripheral blood increase due to reactive causes not involving bone marrow. Although infection is the most common cause, some malignancies may also be seen. Pulmonary, nasopharyngeal, GIS and GUS malignities are the most malign diseases in LR (3). White cell count in our case at time of presentation was $100,000 \mathrm{~mm} 3$, subsequently rising to 127,000 
$\mathrm{mm}^{3}$. No infection was determined in this case. SCC was comorbid with LR, a rare combination despite its association with malign diseases.

In order to make a diagnosis of paraneoplastic LR, secondary causes such as infection, corticosteroid use, severe hemorrhage and bone or bone marrow metastasis have to be excluded. Another possible cause of leukocytosis in malignities is tumor infection and bone marrow infiltration (4). Paraneoplastic LR was suspected in our case when leukocytosis failed to improve following tumor infection treatment, and secondary causes such as drug use and hemolysis were not present. No bone or bone marrow infiltration was identified at bone marrow biopsy.

Probable causes of LR include intoxication, sudden hemolysis and severe hemorrhage. Our patient had no history of drug use and no findings of hemorrhage, and INR and platelet values were normal. Other potential causes of LR such as intoxication, sudden hemolysis and severe hemorrhage were excluded. One retrospective study from China reported that the most common cause of leukocytosis in bone malignities was paraneoplastic LR(4). Our patient had no infection findings, the most common cause of LR. Paraneoplastic LR was diagnosed.

The mechanism whereby leukocytosis develops in malignancies is still the subject of research (5) However, some tumor products such as granulopoietic factors and interleukins are known to be involved (6-8). Leukocyte numbers have been shown to be correlated with serum and urine levels, products of tumor origin that increase in proportion to tumor burden $(3,6)$. However, we were unable to measure tumor products such as G-CSF due to technical reasons.

Hypercalcemia may be seen in paraneoplastic LR. Hypercalcemia in our case was attributed to paraneoplasia. Hydration and forced diuresis were at normal levels during monitoring. Paraneoplastic LR should be considered when infection has been excluded in clinically stable patients with a high tumor burden (9). Our patient was clinically stable with a 
massive tumor. Paraneoplastic LR can be controlled by effective cancer treatment and reducing the tumor burden (4). Our patient had an high initial tumor burden and a leukocyte count of $127,000 \mathrm{~mm}^{3}$ decreasing to $15,000 \mathrm{~mm}^{3}$ when the mass decreased in size following debulking surgery and radiotherapy.

Patients with PLR are often metastatic and resistant to treatment, and prognosis is poor. Insufficient response to debulking surgery and radiotherapy in our case, the presence of hepatic and adrenal metastasis and mortality on the $8^{\text {th }}$ week of hospitalization support this idea.

In conclusion, SCC with paraneoplastic LR is an exceedingly rare combination. This case report was intended to remind physicians of the condition. 


\section{REFERENCES}

1. JT C, TD C. Disorders of Phagocyte Function and Number. In: R H, EJ BJ, SJ S, B F, editors. Hematology Basic Principles and Practice Philadelphia: Churchill; 2000. p. $720-62$.

2. McKee LC, Jr. Excess leukocytosis (leukemoid reactions) associated with malignant diseases. Southern medical journal. 1985; 78: 1475-82.

3. Robinson WA. Granulocytosis in neoplasia. Annals of the New York Academy of Sciences. 1974; 230: 212-8.

4. Ma X, Li G, Cai Z, Sun W, Liu J, Zhang F. Leukemoid reaction in malignant bone tumor patients - a retrospective, single-institution study. European review for medical and pharmacological sciences. 2012; 16: 1895-9.

5. Schniewind B, Christgen M, Hauschild A, Kurdow R, Kalthoff H, Klomp HJ. Paraneoplastic leukemoid reaction and rapid progression in a patient with malignant melanoma: establishment of KT293, a novel G-CSF-secreting melanoma cell line. Cancer biology \& therapy. 2005; 4: 23-7.

6. Cvitkovic E, Bachouchi M, Boussen H, Busson P, Rousselet G, Mahjoubi R, et al. Leukemoid reaction, bone marrow invasion, fever of unknown origin, and metastatic pattern in the natural history of advanced undifferentiated carcinoma of nasopharyngeal type: a review of 255 consecutive cases. Journal of clinical oncology : official journal of the American Society of Clinical Oncology. 1993; 11: 2434-42.

7. Fahey RJ. Unusual leukocyte responses in primary carcinoma of the lung. Cancer. 1951; 4: 930-5.

8. Donati RM, Mc CJ, Lange RD, Gallagher NI. Erythrocythemia and neoplastic tumors. Annals of internal medicine. 1963; 58: 47-55. 
9. Lin CT, Hsu KF, Jao SW, Chen TW, Yu JC, Hsieh CB, et al. Leukemoid reaction with metachronous tumors. Revista espanola de enfermedades digestivas: organo oficial de la Sociedad Espanola de Patologia Digestiva. 2011; 103: 384-5. 